\title{
DIAGNOSE NUTRICIONAL DA CANA-DE-ACÚCAR EM CAMPOS DOS GOYTACAZES (RJ) $)^{(1)}$
}

\author{
R. A. REIS J R. ${ }^{(2)} \&$ P. H. MONNERAT ${ }^{(3)}$
}

\begin{abstract}
RESUMO
Este trabalho objetivou avaliar o estado nutricional de lavouras canaviei ras em Campos dos Goytacazes (RJ) por meio de teores adequados e do Sistema Integrado de Diagnose e Recomendação (DRIS), bem como comparar a diagnose nutricional de padrões descritos na literatura com a diagnose obtida de padrões calibrados regionalmente. Foram coletadas 126 amostras foliares de cana-deaçúcar aos quatro meses de idade das plantas. Essas amostras foram analisadas qui mi camente para N, P, K, Ca, Mg, S, Cu, Mn e Zn, cujos teores foram comparados com: (a) os teores considerados adequados pela literatura, (b) os teores médios de canaviais de alta produtividade localizados em Campos dos Goytacazes e (c) os teores adequados obtidos do DRIS, determinando-se a freqüência com que o teor de cada nutriente foi inferior aos padrões mencionados. As amostras coletadas foram submetidas à diagnose por meio do DRIS, determinando-se a freqüência com que o índice DRIS de cada nutriente apresentou valor negativo e a freqüência com que o índice DRIS de cada nutriente foi o mais negativo dentro de cada amostra. Padrões de diagnose nutricional desenvolvidos em locais distintos forneceram diagnósticos nutricionais diferentes na cana-deaçúcar. As diagnoses nutricionais obtidas de padrões cali brados regionalmente (teores adequados e DRIS) indicaram K, P e S como os principais nutrientes limitantes, enquanto as diagnoses nutricionais de padrões descritos na literatura indicaram N, Zn e Cu como os principais nutrientes limitantes. A calibração regional dos padrões de diagnose da cana-de-açúcar é de grande importância para garantir o sucesso da avaliação do estado nutricional da cana-de-açúcar em Campos dos Goytacazes (RJ).
\end{abstract}

Termos de indexação: Sistema Integrado de Diagnose e Recomendação, DRIS, faixas de suficiência.

\footnotetext{
(1) Parte da Tese de Doutorado do primeiro autor, apresentada à Universidade Estadual do Norte Fluminense - UENF. Parte de trabal ho premiado durante o FertBI 098. Recebido para publicação em setembro de 2000 e aprovado em dezembro de 2001.

(2) Pesquisador, Setor de Fertilidade do Solo e Nutrição Mineral de Plantas, Fundação Chapadão. Rod. MS 306, Km 05, Caixa Postal 39, CEP 79560-000 Chapadão do Sul (MS). E-mail: reisjr@hotmail.com

(3) Professor do Centro de Ciências e Tecnologias Agropecuárias, Universidade Estadual do Norte Fluminense - UENF. CEP 28015-620 Campos dos Goytacazes (RJ ). E-mail: monerat@ienf.br
} 


\title{
SUMMARY: NUTRITIONAL DIAGNOSIS OF SUGARCANE IN CAMPOS DOS GOYTACAZES (RJ - BRAZIL)
}

\begin{abstract}
The present study was conducted to evaluate the nutritional status of sugarcane in Campos dos Goytacazes, Rio deJ aneiro, Brazil, by means of sufficiency rangeand Diagnosis and Recommendation I ntegrated System (DRIS). Fol iar samples werecollected in commercial sugarcane fields to evaluate the N, P, K, Ca, Mg, S, Cu, Mn and Zn concentrations. These foliar contents were compared to: (a) sufficiency rangedescribed in theliterature, (b) average fol iar concentration from a high-yiel ding group cultivated in Campos dos Goytacazes and (c) adequate foliar concentrations obtained with DRIS. The frequency of cases when the fol iar nutrient concentration was bel ow these criteria was determined. DRIS indices were calculated for all the samples collected. The frequency of cases when the DRIS indices for each nutrient were negative and when the DRIS indices of each nutrient were the most negative in each sample were determined. Nutritional standards established in different regi ons showed different nutritional diagnoses in thesugarcane. Nutritional diagnoses with local standards showed K, P and S as themost limiting nutrient, whilenutritional diagnoses with standards described in literatureshowed N, Zn and Cu as themost limiting. Thelocal calibration of the nutritional standards is important to obtain a successful nutritional diagnosis of sugarcanein Campos dos Goytacazes (RJ )/ Brazil.
\end{abstract}

Index terms: Diagnosis and Recommendation Integrated System, DRIS, sufficiency range.

\section{INTRODUÇÃO}

O Brasil col he aproximadamente 340 milhões de toneladas de cana-de-açúcar (FAO, 1999) em quase cinco milhões de hectares (IBGE, 1999), produzindo mais de 12 bilhões de litros de álcool e 13 milhões de toneladas de açúcar (AGRIANUAL, 1998). No estado do Rio de J aneiro, a região nortefluminense, especialmente o município de Campos dos Goytacazes, destaca-se como um pól o da cultura da cana-de-açúcar. A adubação da cana-de-açúcar é fator de alta importância para o aumento de produtividadee representa até $30 \%$ dos seus custos de produção (Zambello J r. et al., 1981). Assim, é importante o uso de métodos que real menteavaliem e calibrem a quantidade de fertilizantes usada nesta cultura, permitindo uso de adubações racionais que visem a aumentos de produtividade e evitem desperdícios de adubos.

O planejamento, a avaliação e a calibração da adubação das culturas podem ser realizados por meio da diagnose nutricional de plantas. Dentre os métodos de diagnose nutricional, destaca-se o Sistema Integrado de Diagnose e Recomendação (DRIS). O DRIS, desenvolvido por Beaufils (1973), é um sistema de interpretação deresultados deanálise de tecidos vegetais (Peverill, 1993) de caráter holístico. Segundo Sumner (1977), Meldal-J ohnsen \& Sumner (1980), J ones J r. (1993) e Bailey et al. (1997), o DRIS foi desenvolvido não só para for necer uma diagnose válida independentemente da idade ou órgão da planta amostrado, mas também para classificar os nutrientes na sua ordem de limitação ao crescimento e desenvolvimento das plantas, permitindo o uso universal das normas DRIS (Sumner, 1979).

O DRIS apresenta vantagem e desvantagens. A vantagem consiste em identificar alguns casos em que a produção está limitada por desequilíbrio nutricional, mesmo quando nenhum dos nutrientes está abaixo de seu nível crítico (Baldock \& Schulte, 1996). As desvantagens do DRIS são: complexidade do método (Bal dock \& Schulte, 1996), não-indicação da probabilidade de resposta à adição do nutriente consideradolimitante (Hallmark \& Beverly, 1991) e dependência entre os índices, ou seja, o teor de um nutriente pode influir na interpretação de outro nutriente (Baldock \& Schulte, 1996).

O DRIS baseia-se no cálculo de um índice para cada nutriente, comparando-se as relações entre um nutriente e cada um dos demais nutrientes na amostra sob diagnose com as relações envolvendo esse mesmo nutriente em uma população de alta produtividade. O índice DRIS de um nutriente consiste na média dos desvios das relações que contêm determinado nutriente em relação a seus respectivos valores ótimos (Bailey et al., 1997). Cada relação entre nutrientes na população de alta produtividade constitui uma norma DRIS etem sua respectiva média e coeficiente de variação. Índice DRIS negativo indica que o nutriente está abaixo do nível ótimo; quando positivo, indica que o nutriente está acima do nível ótimo (Baldock \& Schulte, 1996). Se oíndice DRIS de um nutriente é igual a zero, este elemento é considerado estar em perfeito equilíbrio com os outros nutrientes (Payne et al., 1990). 
Osíndices dos nutrientes em uma amostra podem variar de positivos a negativos, mas o somatório destes índices sempre será igual a zero (Elwali \& Gascho, 1983). O somatório dos valores absolutos destes índices forma o Índice de Equilíbrio Nutricional (IEN) (Baldock \& Schulte, 1996), que expressa o equilíbrio nutricional da lavoura amostrada. Quanto menor o IEN, menor será o desequilíbrioentrenutrientes (Snyder \& Kretschmer, 1988).

Este trabalho objetivou avaliar o estado nutricional delavouras canavieiras em Campos dos Goytacazes (RJ ) por meio de teores adequados e do Sistema I ntegrado de Diagnose e Recomendação (DRIS) ecomparar a diagnose nutricional de padrões descritos na literatura com a diagnose obtida de padrões calibrados regionalmente.

\section{MATERIAL E MÉTODOS}

Entre agosto/96 e fevereiro/97, foram col etadas 126 amostras foliares de cana-de-açúcar (canaplanta e cana-soca), da posição +3 , de plantas com quatro meses de idade, das variedades CB 45-3, RB 72-454, RB73-9735 eSP70-1143 no município de Campos dos Goytacazes (RJ ). Cada amostra foi constituída de 15 fol has coletadas al eatoriamenteem uma área de aproximadamente um hectare.

Das fol has amostradas, foram utilizados na análise química os 20 centímetros medianos, descartando-se a nervura central. Estas amostras foram submetidas à secagem em estufa a $70^{\circ} \mathrm{C}$ com circulação forçada dear por 72 h e moí das em moinho tipo Wiley (com peneiras de 20 mesh). Foram analisados os teores de $\mathrm{N}$-orgânico, pelo método de Nessler (J ackson, 1965), após a digestão do material $\operatorname{seco}(0,1 \mathrm{~g}) \mathrm{com} \mathrm{H}_{2} \mathrm{SO}_{4}$ concentrado $(1,5 \mathrm{~mL})$ e $\mathrm{H}_{2} \mathrm{O}_{2}$ $30 \%$ (1 mL); o teor de fósforo, colorimetricamente pelo método do molibdato; o teor de potássio, por espectrofotometria atômica de emissão de chama; os teores de $\mathrm{Ca}, \mathrm{Mg}, \mathrm{Mn}, \mathrm{Zn}$ e $\mathrm{Cu}$, por espectrofotometria de absorção atômica, e o teor de enxofre, por turbidimetria após digestão do material seco $(0,5 \mathrm{~g})$ com ácido nítrico $65 \%$ (4 mL) eácido perclórico $70 \%$ (2 mL).

Dados de produtividade for am registrados nestes locais amostrados quando estes atingiram o ponto de colheita, formando um banco de dados que relacionou produtividade e teores foliares. Este banco de dados foi dividido em grupos de alta ( $\geq 75 \mathrm{Mg} \mathrm{ha}^{-1}$ ) e baixa produtividade ( $\left.<75 \mathrm{Mg} \mathrm{ha}^{-1}\right)$ e utilizado para gerar as normas DRIS para a canade-açúcar, conforme descrito em Reis J r. (1999).

Os teores dos nutrientes do banco de dados foram comparados com: (a) os teores considerados adequados pela literatura (Quadros 1 e 2), (b) com os teores médios de canaviais de alta produtividade usados para gerar as normas DRIS, descritas em Reis J r. (1999) (Quadro 3), e (c) com os teores adequados cal culados para obter oíndice DRIS nulo proposto por ReisJ r. (1999) (Quadro 3), determinandose a freqüência com que o teor de cada nutriente foi inferior ao dos supracitados.

A partir dos teores do banco de dados, foram calculados os índices DRIS com as normas DRIS descritas em Reis J r. (1999) e constante de sensibilidade $(\mathrm{k})$ igual a 10 , determinando-se a freqüência com que o índice DRIS de cada nutriente apresentou valor positivo e negativo e a freqüência com que o índice DRIS de cada nutriente foi o mais positivo e negativo dentro de cada amostra avaliada.

Os dados foram analisados por meio deestatística descritiva.

Quadro 1. Teores de macronutrientes na matéria seca da folha +3 da cana-de-açúcar considerados adequados para a cana-de-açúcar e suas respectivas épocas de amostragem

\begin{tabular}{|c|c|c|c|c|c|c|c|}
\hline & $\begin{array}{c}\text { É poca de } \\
\text { amostragem }\end{array}$ & $\mathbf{N}$ & $\mathbf{P}$ & $K$ & $\mathrm{Ca}$ & Mg & $\mathbf{S}$ \\
\hline & mês & 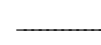 & 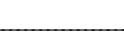 & - & & & - \\
\hline Cana-planta & $4^{(1)}$ & $19-21$ & $2,0-2,4$ & $11-13$ & $8-10$ & $2-3$ & $2,5-3,0$ \\
\hline Cana-soca & $4^{(1)}$ & $20-22$ & $1,8-2,0$ & 13-15 & $5-7$ & $2-3$ & $2,5-3,0$ \\
\hline Cana-planta & $4^{(2)}$ & 23 & $1,7-1,8$ & $8,8-10$ & - & - & - \\
\hline Cana-soca & $4(2)$ & $20-22$ & $2,1-2,2$ & $10-15$ & - & - & - \\
\hline Cana-planta & $3(3)$ & $20-22$ & $2,0-2,4$ & $11-13$ & $9-11$ & $2-3$ & $2-3$ \\
\hline Cana-soca & $3^{(3)}$ & $20-22$ & $2,0-2,4$ & $11-13$ & $9-11$ & $2-3$ & $2-3$ \\
\hline
\end{tabular}

(1) Malavolta et al. (1997). (2) Orlando Filho \& Campos (1975a,b). ${ }^{(3)}$ Orlando Filho \& Haag (1976). 
Quadro 2. Teores de micronutrientes na matéria seca da folha +3 da cana-de-açúcar considerados adequados para a cana-de-açúcar e suas respectivas épocas de amostragem

\begin{tabular}{|c|c|c|c|c|c|c|}
\hline & $\begin{array}{c}\text { É poca de } \\
\text { amostragem }\end{array}$ & B & $\mathrm{Cu}$ & Zn & $M n$ & $\mathbf{F e}$ \\
\hline & mês & 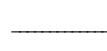 &  & $\mathrm{mg} \mathrm{kg}^{-1}$ & 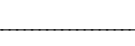 & - \\
\hline Cana-planta & $4^{(1)}$ & - & - & $15-26$ & - & - \\
\hline Cana-soca & $4^{(1)}$ & - & - & $23-24$ & - & - \\
\hline Cana-planta & $4(2)$ & - & $8-10$ & - & - & $245-507$ \\
\hline Cana-soca & $4^{(2)}$ & - & $8-11$ & - & - & $86-140$ \\
\hline Cana-planta & $4^{(3)}$ & - & $9,5-13,7$ & - & - & - \\
\hline Cana-soca & $4^{(3)}$ & - & $11,5-12,7$ & - & - & - \\
\hline Cana-planta & $4^{(4)}$ & - & - & - & - & $160-335$ \\
\hline Cana-soca & $4^{(4)}$ & - & - & - & - & 174-392 \\
\hline Cana-planta & $-(5)$ & - & - & - & $192-249$ & - \\
\hline Cana-soca & $-(5)$ & - & - & - & $138-222$ & - \\
\hline Cana-planta & $4^{(6)}$ & $15-50$ & $8-10$ & $25-30$ & $100-250$ & $200-500$ \\
\hline Cana-soca & $4^{(6)}$ & - & $8-10$ & $25-50$ & $50-125$ & $80-150$ \\
\hline
\end{tabular}

Quadro 3. Teores médios de nutrientes obtidos em canaviais de alta produtividade localizados em Campos dos Goytacazes (TMA) e teores adequados propostos por Reis J r. (1999) (TA)

\begin{tabular}{|c|c|c|c|c|c|c|c|c|c|}
\hline & $\mathbf{N}$ & $\mathbf{P}$ & K & $\mathrm{Ca}$ & Mg & $\mathbf{S}$ & $\mathbf{C u}$ & Mn & Zn \\
\hline & & & 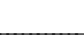 & & 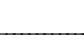 & 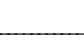 &  & $\mathrm{mg} \mathrm{kg}$ & 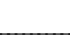 \\
\hline TMA & 14,9 & 2,12 & 12,4 & 3,44 & 2,68 & 1,89 & 5,00 & 74,4 & 14,3 \\
\hline $\mathrm{TA}$ & 13,4 & 1,91 & 12,2 & 2,99 & 2,15 & 1,61 & 4,48 & 67,8 & 11,7 \\
\hline
\end{tabular}

Folha amostrada (+3). É poca de amostragem (4 meses de idade).

\section{RESULTADOS E DISCUSSÃO}

Nitrogênio (96,8 \% das amostras), zinco (99,2 \%), cobre $(98,4 \%)$, enxofre $(82,5 \%)$ e cálcio $(80,4 \%)$ destacaram-seentre os nutrientes queapresentaram teores, nas amostras avaliadas, inferiores aos listados nos quadros 1 e2, indicando, a princípio, que estes nutrientes estariam limitando a produção da cana-de-açúcar. Todavia, ao utilizar critérios estabelecidos em Campos dos Goytacazes para interpretar a análise química das amostras, outros nutrientes destacaram-se, tais como: fósforo, potássio e enxofre (Quadro 4).

Quando se consideraram os teores médios dos canaviais de alta produtividade, utilizados para gerar as normas DRIS para a cana-de-açúcar, e os teores adequados, cal culados para obter índiceDRIS nulo na interpretação dos resultados da análise química das 126 amostras col etadas neste trabal ho, $\mathrm{K}, \mathrm{P}$ eS foram os nutrientes que apresentaram teores inferiores aos demais em maior freqüência.

Ao levar em consideração o sinal do índice DRIS cal culado para os nutrientes das amostras do banco de dados, verificou-se que potássio $(72,2 \%$ das amostras), fósforo (58,7 \%) e enxofre $(57,1 \%)$ destacaram-se, novamente, como os nutrientes que apresentavam índices negativos em mai or freqüência (Quadro 5). Considerando, contudo, qual onutriente, dentro de cada amostra fol iar, mostrava oíndice mais negativo e, a princípio, o mais limitante, notou-se que enxofre ( $21,4 \%)$, cobre $(21,4 \%)$ e zinco ( $11,9 \%)$ destacaram-se, revelando ser a limitação por estes nutrientes maior quea limitação por fósforo epotássio.

Os teores médios das lavouras de alta produtividade ( $\geq 75 \mathrm{Mg} \mathrm{ha}^{-1}$ ) e os teores adequados propostos por Reis J r. (1999) para N, Ca e Cu 
Quadro 4. Número e freqüência de amostras que apresentaram nutrientes com teores inferiores aos considerados adequados pela literatura (A), aos teores médios de nutrientes obtidos em canaviais de alta produtividade localizados em Campos dos Goytacazes (TMA) e aos teores adequados propostos por Reis J r. (1999) (TA)

\begin{tabular}{lrcccccccc}
\hline & N-org & P & K & Ca & Mg & S & Cu & Mn & Zn \\
\hline A & $122(96,8)$ & $56(44,4)$ & $12(33,3)$ & $101(80,2)$ & $34(27,0)$ & $104(82,6)$ & $124(98,4)$ & $22(17,5)$ & $125(9,2)$ \\
TMA & $69(54,8)$ & $96(76,2)$ & $107(84,9)$ & $49(38,9)$ & $82(65,1)$ & $93(73,8)$ & $68(54,0)$ & $59(46,8)$ & $80(63,5)$ \\
TA & $32(25,4)$ & $72(57,1)$ & $103(81,7)$ & $27(21,4)$ & $41(32,5)$ & $66(52,4)$ & $53(42,1)$ & $50(39,7)$ & $55(43,7)$
\end{tabular}

Obs.: número total de amostras $=126$. Números entre parênteses representam a freqüência, em \%.

Quadro 5. Número e freqüência de amostras que apresentaram índices DRIS negativos (I-) e positivos (I +) para os nutrientes estudados e número e freqüência de amostras que apresentaram o menor $(<$ I) e o maior índice DRIS (>I) dentro de cada amostra para os nutrientes estudados

\begin{tabular}{cccccccccc}
\hline & \multicolumn{10}{c}{ Índice DRIS } \\
\cline { 2 - 10 } & I N & IP & I & ICa & I Mg & IS & IC U & I Mn & IZn \\
\hline $1-$ & $39(31,0)$ & $74(58,7)$ & $91(72,2)$ & $28(22,2)$ & $52(41,3)$ & $72(57,1)$ & $61(48,4)$ & $49(38,9)$ & $65(51,6)$ \\
$1+$ & $87(69,0)$ & $52(41,3)$ & $35(27,8)$ & $98(77,8)$ & $74(58,7)$ & $54(42,9)$ & $65(51,6)$ & $77(61,1)$ & $61(48,4)$ \\
$<1$ & $8(6,3)$ & $9(7,1)$ & $14(11,1)$ & $11(8,7)$ & $5(4,0)$ & $27(21,4)$ & $27(21,4)$ & $10(7,9)$ & $15(11,9)$ \\
$>1$ & $14(11,1)$ & $3(2,4)$ & $3(2,4)$ & $32(25,4)$ & $7(5,6)$ & $6(4,8)$ & $19(15,1)$ & $31(24,6)$ & $11(8,7)$
\end{tabular}

Obs.: número total de amostras = 126. Números entre parênteses representam a freqüência, em \%.

(Quadro 3) foram inferiores aos teores considerados adequados na literatura (Quadros 1 e 2). Desta forma, ao avaliar o estado nutricional das lavouras de alta produtividade com os padrões descritos nos quadros 1 e 2, seria diagnosticado limitação nutricional por parte de N, Ca e Cu. Pel o fato de as lavouras de al tas produtividades não apresentarem limitação nutricional, conclui-se que os padrões de diagnose nutricional descritos nos quadros 1 e 2 não são indicados para a diagnose nutricional das lavouras de cana-de-açúcar em Campos dos Goytacazes, pelo menos para os patamares de produtividade encontrados nesta região. Isto reforça a necessidade de calibração local/regional de padrões utilizados na diagnosenutricional da cana-de-açúcar.

É possível que os critérios estabelecidos regionalmente forneçam maior confiança à diagnose nutricional, visto queos trabal hos queestabel eceram os teores considerados adequados pela literatura (Quadros 1e2) foram, em sua maioria, desenvolvidos sob condições de solo e clima diferentes das encontradas em Campos dos Goytacazes.

Como exemplo disto, destaca-se que os teores médios de nitrogênio, cálcio, enxofre, cobre e zinco dos canaviais de alta produtividade, usados para estabelecer as normas DRIS, foram inferiores aos considerados adequados nos quadros 1 e 2 . Isto reforça a teoria de que os critérios regionais estabelecidos neste trabal ho possam dar mais confiança à diagnose nutricional na cana-de-açúcar.

Ao levar em consideração principalmente os critérios estabel ecidos em Campos dos Goytacazes, fósforo, potássio, enxofre, cobre e zinco seriam os nutrientes que possivel mente estariam limitando a produção da cana-de-açúcar. O potássio tem papel reconhecido na síntese de açúcares e é o nutriente mais exportado pela cultura da cana-de-açúcar, enquanto o fósforo apresenta a característica de não propiciar, de modo geral, aumentos de produtividade na cana-soca, sendo até suprimido das adubações de soqueira. Assim, cuidados para uma adequada reposição do potássio exportado na col heita da canade-açúcar, bem como cuidados para uma correta adubação fosfatada no plantio desta cultura, devem ser tomados.

Segundo Orlando Filho et al. (1994), cobree zinco são os micronutrientes mais limitantes para a cultura da cana-de-açúcar no Brasil, e o constante uso de adubações que continham basicamente nitrogênio e potássio nas lavouras instaladas em Campos dos Goytacazes pode ter contribuído para o surgimento da limitação por parte destes nutrientes. A exemplo dos micronutrientes, o uso efetivo do enxofre na prática de adubação da cana-de-açúcar 
não é comum e, com o uso de fertilizantes cada vez mais concentrados, a partici pação deste nutriente na adubação da cana-de-açúcar vem sendo suprimida.

\section{CONCLUSÕES}

1. As diagnoses nutricionais de padrões calibrados regional mente (teores adequados eDRIS) indicaram $\mathrm{K}$, $\mathrm{P}$ e $\mathrm{S}$ como os principais nutrientes limitantes, enquanto as diagnoses nutricionais de padrões descritos na literatura indicaram N, Zn e Cu como os principais nutrientes limitantes.

2. A cal ibração regional dos padrões de diagnose da cana-de-açúcar é de grande importância para garantir o sucesso da avaliação do estado nutricional da cana-de-açúcar em Campos dos Goytacazes (RJ ).

\section{AGRADECIMENTOS}

À Usina Santa Cruz Ltda., pelo apoio prestado durante a realização deste trabal ho.

\section{LITERATURA CITADA}

AGRIANUAL 98. São Paulo, 1998. p.176.

BAILEY, J.S.; BEATTIE, J.A.M. \& KILPATRICK, D.J. The diagnosis and recommendation integrated system (DRIS) for diagnosing the nutrient status of grassland swards: I. Model establishment. Plant Soil, 197:127-135, 1997.

BALDOCK, J.O. \& SCHULTE, E.E. Plant analysis with standardized scores combines DRIS and sufficiency range approaches for corn. Agron. J., 88:448-456, 1996.

BEAUFILS, E.R. Diagnosis and recommendation integrated system (DRIS). Pietermaritizburg, University of Natal, 1973. 132p. (Soil Science Bulletin, 1)

ELWALI, A.M.O. \& GASCHO, G.J . Sugarcane response to $P, K$ and DRIS corrective treatments on Florida Histosols. Agron. J., 75:79-83, 1983.

HALLMARK, W.B. \& BEVERLY, R.B. Review - an update in the use of the Diagnosis and Recommendation Integrated System. J. Fertil., 8:74-88, 1991.

FAO STAT. Database gateway. 1999. (http: \\www.fao.org)

INSTITUTO BRASILEIRO DE GEOGRAFIA E ESTATÍSTICA - IBGE. Sistema IBGE de Recuperação Automática SIDRA 97. 1999. Capturado em 19 de janeiro de 1999 (http:\\www.sidra.ibge.gov.br)

J ACKSON, M.L. Soil chemical analysis. New J ersey, Prentice Hall, 1965. 498p.

MALAVOLTA, E.; VITTI, G.C. \& OLIVEIRA, S.A. Avaliação do estado nutricional das plantas - princípios e aplicações. Piracicaba, POTAFOS, 1997. 319p.
MELDAL-J OHNSEN, A. \& SUMNER, M.E. Foliar diagnostic norms for potatoes. J. Plant Nutr., 2:569-576, 1980.

ORLANDO FILHO, J. \& CAMPOS, H. Número ideal de fol has para diagnose foliar em cana-de-açúcar (cana planta). Brasil Açuc., 85:10-17, 1975a.

ORLANDO FILHO, J. \& CAMPOS, H. Número ideal de folhas para diagnose foliar em cana-de-açúcar (soqueira). Brasil Açuc., 85:23-29, 1975b.

ORLANDO FILHO, J . \& HAAG, H.P. Levantamento do estado nutricional de $\mathrm{N}, \mathrm{P}, \mathrm{K}, \mathrm{Ca}, \mathrm{Mg}$ e $\mathrm{S}$ em 16 variedades da cana-de-açúcar (Saccharum spp) pela análise foliar. Brasil Açuc., 88:11-27, 1976.

ORLANDO FILHO, J \& Z ZAMBELLO J r., E. Diagnose foliar de $\mathrm{Cu}, \mathrm{Fe}, \mathrm{Mn}$ e Zn em 16 variedades de cana-de-açúcar (Saccahrum spp) cultivados em diferentes grandes grupos de solos. Brasil Açuc., 90:28-37, 1977.

ORLANDO FILHO, J .; ZAMBELLO J r., E. \& HAAG, H.P. Absorção de Zn pela cana-de-açúcar, variedade CB 41-76, em três solos no Estado de São Paulo. Brasil Açuc., 96:2130, 1980a.

ORLANDO FILHO, J.; ZAMBELLO J r., E. \& HAAG, H.P. Acumulação de $\mathrm{Mn}$ pela parte aérea da cana-de-açúcar em função da idade. Piracicaba, PLANALSUCAR, 1980c. p.330. (Boletim Técnico, 2)

ORLANDO FILHO, J.; ZAMBELLO J r., E. \& HAAG, H.P. Influência do solo na absorção de Cu pela cana-de-açúcar, variedade CB 41-76, em função da idade. In: CONGRESSO NACIONAL DA SOCIEDADE DE TÉCNICOS AÇUCAREIROS DO BRASIL, 13., Maceió, 1980. Anais. Maceió, STAB, 1980b. p.304-314.

ORLANDO FILHO,J .; MACEDO, N. \& TOKESHI, H. Seja doutor do seu canavial. Inf. Agron., 67:1-16, 1994.

ORLANDO FILHO, J .;ZAMBELLOJ r, E.\& HAAG, H.P. Marcha de absorção de Fe pela cana-de-açúcar em solos do Estado de São Paulo. Ann. ESALQ, 36:387-402, 1979.

PAYNE, G.G.; RECHCIGL, J.E. \& STEPHENSON, R.L. Devel opment of Diagnosis and Recommendation I ntegrated System norms for Bahiagrass. Agron. J ., 82:930-930, 1990.

PEVERILL, K.I. Soil testing and plant analysis in Australia. Aust. J. Exp. Agric., 33:963-71, 1993.

REIS J r., R.A. Diagnose nutricional da cana-de-açúcar com o uso do Sistema Integrado de Diagnose e Recomendação (DRIS). Campos dos Goytacazes, Universidade Estadual do Norte Fluminense, 1999. 141p. (Tese de Doutorado)

SNYDER, G.H. \& KRETSCHMER J r., A.E. A DRIS analysis for Bahiagrass pastures. Soil Crop Sci. Soc. Florida Proc., 47:56-59, 1988.

SUMNER, M.E. Effect of corn leaf sampled on N, P, K, Ca and $\mathrm{Mg}$ content and calculated DRIS indices. Comm. Soil Sci. Plant Anal., 8:269-280, 1977.

SUMNER, M.E. Interpretation of foliar analysis for diagnostic purposes. Agron. J., 71:343-348, 1979.

ZAMBELLO J r., E.; HAAG, H.P. \& ORLANDO FILHO, J. Aplicação do sistema integrado de diagnose e recomendação (DRIS) em soqueiras de cana-de-açúcar para diferentes épocas de amostragem foliar. Piracicaba, PLANALSUCAR, 1981. p.32. (Boletim Técnico, 3) 